\title{
Hall and Rotation Effects on Radiating and Reacting MHD Flow past an Accelerated Permeable Plate with Soret and Dufour Effects
}

\author{
Paul Matao*, Bijjula Prabhakar Reddy and Jefta Sunzu \\ Department of Mathematics and Statistics, College of Natural and Mathematical Sciences, \\ The University of Dodoma, Dodoma, Tanzania
}

('Corresponding author's e-mail: paul.matao@udom.ac.tz)

Received: 1 October 2020, Revised: 28 May 2021, Accepted: 1 June 2021

\begin{abstract}
This article investigates numerically the effects of Hall current and rotation effects on radiating and chemically reacting unsteady MHD natural convection flow past an accelerated infinite vertical permeable plate in the presence of Soret and Dufour effects. The dimensionless coupled non-linear governing partial differential equations of the problem are solved numerically by employing finite element method. The influence of various physical parameters influencing the flow on the primary velocity, secondary velocity, temperature and the species concentration are displayed graphically whilst the numerical results of the primary skin friction, secondary skin-friction, Nusselt number and the Sherwood number are presented in tabular form. Results reveals that magnetic parameter, radiation parameter and chemical reaction rate tends to depreciate both primary and secondary velocity components whilst Hall, Soret and Dufour effects have reverse trend. Rotation parameter tends to retard fluid flow in the primary flow direction and accelerate fluid flow in the secondary flow direction. Thermal boundary layer thickness decreases with increasing radiation parameter whilst the reverse trend is noticed with increasing Dufour effect. Thermal diffusion effect causes to improve concentration boundary layer thickness whilst chemical reaction rate has reverse impact. These parameters have similar effect on the primary and secondary skin-frictions whilst opposite effect was noticed on the Nusselt and Sherwood numbers. This model problem finds an important in engineering and industrial application such as MHD generators, food processing, heat exchangers devices and internal rotation rate of the sun.
\end{abstract}

Keywords: Dufour number, Hall current, Magnetic field, Rotation, Soret number

\section{Introduction}

The study of thermal radiation and chemical reaction effects on MHD has become significant during the last decades due to its numerous industrial, scientific and engineering applications, such as manufacturing of ceramics, packed-bed catalytic reactors, food processing, underground energy transport, magnetized plasma flows, cooling of nuclear reactors, high speed plasma wind, cosmic jets and stellar systems, heat exchangers devices, petroleum reservoirs, insulation systems, filtration, irrigation pumps and wet bulb thermometers. Because of these significant applications, Makinde [1] studied MHD natural and forced convection interaction with thermal radiation and chemical reaction past a vertical porous plate in a porous medium. Reddy [2] evaluated analytically chemical reaction and thermal radiation effects on MHD mixed convective boundary layer slip flow in a porous medium in the presence of heat source and Ohmic heating. Manjula et al. [3] scrutinized chemical reaction and thermal radiation effects on MHD flow heat and mass transfer over a stretching surface. Unsteady MHD free convection heat and mass transfer in a boundary layer flow past a vertical permeable plate with chemical reaction and thermal radiation were reported by Venkateswarlu and Padma [4]. Thermal radiation and chemical reaction effects on an exponentially accelerated vertical plate with variable temperature and uniform mass diffusion were discussed by Lakshmi and Muthucumaraswamy [5]. Muthucumaraswamy and Sivakumar [6] studied MHD free convection flow with a parabolic starting motion of an infinite isothermal vertical plate in the presence of thermal radiation and chemical reaction. Eegunjobi et al. [7] studied unsteady MHD chemically reacting and radiating mixed convection slip flow past a stretching surface in a porous medium. The effects of radiation on unsteady MHD free convection flow of an incompressible viscous, electrically conducting fluid near an infinite vertical plate with ramped wall temperature and the chemical 
reaction has been discussed by Reddy [8]. Babu et al. [9] presented the effects of chemical reaction and thermal radiation on MHD mixed convection flow over a vertical plate with variable fluid properties. Reddy [10] studied the impact of chemical reaction on a transient MHD mixed convection flow with mass transfer past an impulsively fixed infinite vertical plate under the effect of thermal radiation. Idowu and Sani [11] examined the thermal radiation and chemical reaction effects on unsteady MHD third grade fluid flow between stationary and oscillating plates.

In all the above mentioned studies, the effects of Hall current on the flow are not considered. The Hall current is generated in the flow field when an electrically conducting fluid with low density is exposed to a strong magnetic field. It plays vital role in determining the carrier concentration and flow features as it induces secondary flow in the flow field. The applications of Hall current find it is significant in the Hall accelerators, oil extraction, electronic system, MHD pumps and MHD generators. Siddiqa [12] analyzed the impact of Hall current on MHD natural convection flow with a strong cross magnetic field. Anika [13] studied numerically unsteady MHD flow past an infinite rotating vertical porous plate with heat transfer in the presence of the Hall current. Maguna and Mutua [14] discussed the effect of Hall current on free convection flow and mass transfer past a semi-infinite vertical flat plate. Seth et al. [15] investigated rotation and Hall effects on natural convection flow with heat and mass transfer past a moving vertical plate in the presence of radiation. Sundarnath and Muthucumarsway [16] presented the Hall effects on MHD heat transfer flow past an accelerated horizontal plate relative to a rotating fluid. Sulochana [17] scrutinized the impact of Hall current on unsteady MHD convective flow heat generating and absorbing fluid through porous medium in a rotating parallel plate. Rajput and Kanaujia [18] studied MHD flow past a vertical plate with variable temperature and mass diffusion in the presence of Hall current. Seth et al. [19] examined the effect of Hall current on MHD natural convection heat and mass transfer flow of rotating fluid past a vertical plate with ramped wall temperature. An investigation of unsteady MHD natural convection flow past an infinite vertical plate through a porous medium with exponentially accelerated free-stream in a rotating system with Hall effects was carried out by Seth et al. [20]. Sekhar [21] studied the Hall current, rotation and thermal diffusion effects on MHD free convective heat and mass transfer flow past an accelerated vertical plate through a porous medium. Reddy [22] investigated the effect of Hall current on MHD transient flow past an impulsively started infinite horizontal porous plate in a rotating system. Krishna [23] studied MHD heat and mass transfer rotating flow of second grade fluid past an infinite vertical plate embedded in a uniform porous medium with Hall current. Recently, Krishna [24] studied Hall and ion slip effects on MHD free convective rotating flow bounded by the semi-infinite vertical porous surface.

However, in all the above cited works, the effects of thermal diffusion (Soret) and diffusion thermo (Dufour) on the flow have been neglected from the concentration and energy equations, respectively. The energy flux caused by temperature gradient is called Soret effect and the energy flux caused by concentration gradient is called Dufour effect. These effects are very significant when the temperature and concentration gradients are very high. According to Eckert and Drake [25] cross diffusion effects become important if not dominant in materials processing operations such as magnetic separation of colloids and flow of different densities. Makinde [26] studied the Soret and Dufour effects on MHD mixed convection flow past a vertical plate embedded in porous medium. Srinivasacharya and Ramreddy [27] have analyzed MHD mixed convection heat and mass transfer flow, from an exponentially stretching vertical surface in a quiescent fluid. Subhakar and Gangadhar [28] discussed the Soret and Dufour effects on MHD convective flow of heat and mass transfer over a moving vertical plate with heat generation. Cheng [29] examined free convection boundary layer flow over an arbitrary inclined heated plate in a porous medium with Soret and Dufour effects. The Soret and Dufour effects on mixed convection heat and mass transfer along a vertical plate embedded in a power law fluid in saturated porous medium were studied by Srinivasacharya and Reddy [30]. Seini and Makinde [31] investigated the effects of Soret and Dufour on hydro-magnetic flow past a vertical plate embedded in porous media. The Soret effect on unsteady mixed convection heat and mass transfer flow over an oscillating vertical plate embedded in a porous medium with Newtonian heating in the presence of magnetic field analyzed by Hussanan et al. [32].

The above detailed literature assessment reveals that the problem of rotating, radiating and chemically reacting unsteady MHD natural convection heat and mass transfer flow past an accelerated infinite vertical permeable plate through the porous medium in the presence of Hall current with Soret and Dufour effects is not being discussed. Such problem is very important in the context of rotating MHD generators, structure of the magnetic stars, internal rotation rate of the sun, food processing and in the study of isotope separation in mixtures between gases with very light molecular weight and medium molecular weight. The main objective of the present work is to fill this gap. Hence, it is proposed to 
investigate the effects of Hall current and rotation effects on radiating and reacting unsteady MHD natural convection heat and mass transfer flow past an accelerated infinite vertical permeable plate with Soret and Dufour effects. The dimensionless coupled strongly nonlinear governing partial differential equations of the problem have been solved numerically by using finite element method. The numerical results of primary and secondary velocity components, temperature and species concentration are depicted graphically whilst the primary and secondary skin-frictions, Nusselt and Sherwood numbers are presented in tabular and then discussed.

\section{Materials and methods}

Consider the unsteady MHD natural convection heat and mass transfer flow of a viscous, incompressible and electrically conducting fluid flow past an accelerated infinite vertical permeable plate embedded in a uniform porous medium. The coordinate system $\left(x^{\prime}, y^{\prime}, z^{\prime}\right)$ is chosen in such a way that $x^{\prime}$ - axis is along the plate in skyward direction, $y^{\prime}$ - axis normal to the plane of the plate and $z^{\prime}$ - axis normal to $x^{\prime} y^{\prime}$ - plane (Figure 1). A uniform transverse magnetic field of intensity $B_{0}$ is imposed normal to the plate. The fluid and plate rotate in accord with uniform angular velocity $\Omega^{\prime}$ about $y^{\prime}$ - axis. Initially, at time $t^{\prime} \leq 0$, the fluid and plate are at rest and uniform temperature is maintained at $T_{\infty}^{\prime}$, the species concentration at the surface of the plate and at every point inside the fluid is preserved at uniform concentration $C_{\infty}^{\prime}$. Subsequently, when time $t^{\prime}>0$, the plate starts moving in $x^{\prime}$ - direction with velocity $u^{\prime}=U t^{\prime}$ in its own plane and the temperature and species concentration at the surface of the plate is raised or lowered to $T_{w}^{\prime}$ and $C_{w}^{\prime}$. The plate is an infinite extent in $x^{\prime}$ and $z^{\prime}$ - directions, so all the physical quantities except the pressure are the functions of $y^{\prime}$ and $t^{\prime}$ only. Furthermore, no applied or polarized voltages exist so the effect of polarization of fluid is negligible because magnetic Reynolds number is very small for liquid metals and partially ionized fluids which are commonly used in various industrial processes. Under the above assumptions and taking into account the usual boundary layer approximations, the governing boundary layer equations for this physical situation are derived as;

$$
\begin{aligned}
& \frac{\partial u^{\prime}}{\partial t^{\prime}}+2 \Omega w^{\prime}=v \frac{\partial^{2} u^{\prime}}{\partial y^{\prime 2}}-\frac{\sigma B_{0}^{2}}{\rho\left(1+m^{2}\right)}\left(u^{\prime}+m w^{\prime}\right)+g \beta_{\theta}\left(T^{\prime}-T_{\infty}^{\prime}\right)+g \beta_{c}\left(C^{\prime}-C_{\infty}^{\prime}\right)-\frac{v u^{\prime}}{K^{\prime}} \\
& \frac{\partial w^{\prime}}{\partial t^{\prime}}-2 \Omega u^{\prime}=v \frac{\partial^{2} w^{\prime}}{\partial y^{\prime 2}}+\frac{\sigma B_{0}^{2}}{\rho\left(1+m^{2}\right)}\left(m u^{\prime}-w^{\prime}\right)-\frac{v w^{\prime}}{K^{\prime}} \\
& \frac{\partial T^{\prime}}{\partial t^{\prime}}=\frac{k}{\rho C_{p}} \frac{\partial^{2} T^{\prime}}{\partial y^{\prime 2}}-\frac{1}{\rho C_{p}} \frac{\partial q_{r}^{\prime}}{\partial y^{\prime}}+\frac{D_{M} K_{T}}{C_{s} C_{p}} \frac{\partial^{2} C^{\prime}}{\partial y^{\prime 2}}
\end{aligned}
$$

$$
\frac{\partial C^{\prime}}{\partial t^{\prime}}=D_{M} \frac{\partial^{2} C^{\prime}}{\partial y^{\prime 2}}+\frac{D_{M} K_{T}}{T_{m}} \frac{\partial^{2} T^{\prime}}{\partial y^{\prime 2}}-K_{r}^{\prime}\left(C^{\prime}-C_{\infty}^{\prime}\right)
$$




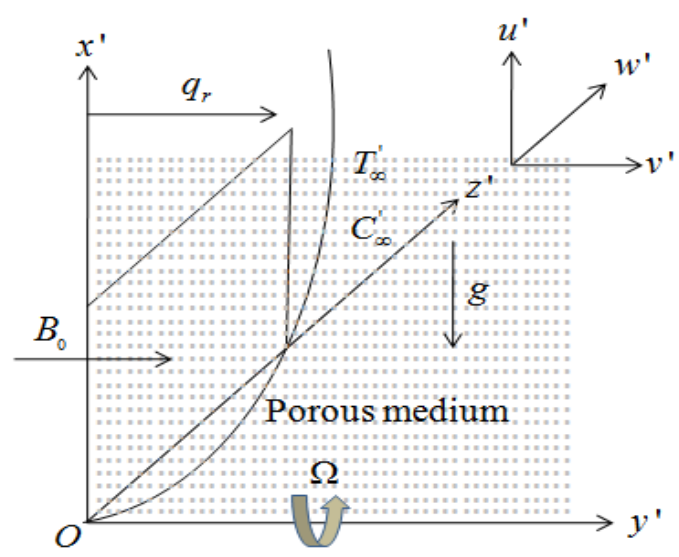

Figure 1 Flow geometry and Coordinate system.

where $u^{\prime}, w^{\prime}, g, \rho, \beta_{\theta}, \beta_{c}, k^{\prime}, C_{p}, C_{s}, \sigma, v, m\left(=\omega_{e} \tau_{e}\right), \omega_{e}, \tau_{e}, D_{M}, K_{T}, T_{m}, C^{\prime}, T^{\prime}, K_{r}^{\prime}, q_{r}^{\prime}$ and $K^{\prime}$ are respectively, the fluid velocity in $x^{\prime}$-direction, fluid velocity in $z^{\prime}$-direction, acceleration due to gravity, the fluid density, the volumetric coefficient of thermal expansion, the volumetric coefficient of concentration expansion, thermal conductivity, specific heat at constant pressure, concentration susceptibility, electrical conductivity, the kinematic viscosity, Hall current parameter, cyclotron frequency, electron collision time, the coefficient of mass diffusivity, the thermal diffusion ratio, the mean fluid temperature, species concentration, the temperature of the fluid, chemical reaction parameter, radiative heat flux vector and permeability of porous medium.

The corresponding initial and boundary conditions flow are;

$u^{\prime}=0, w^{\prime}=0, T^{\prime}=T_{\infty}^{\prime}, C^{\prime}=C_{\infty}^{\prime} \quad$ for all $y^{\prime}$ and $t^{\prime} \leq 0$

$u^{\prime}=U t^{\prime}, w^{\prime}=0, T^{\prime}=T_{w}^{\prime}, C^{\prime}=C_{w}^{\prime}$ at $y^{\prime}=0$ for $t^{\prime}>0$

$u^{\prime} \rightarrow 0, w^{\prime} \rightarrow 0, T^{\prime} \rightarrow T_{\infty}^{\prime}, C^{\prime} \rightarrow C_{\infty}^{\prime} \quad$ as $y^{\prime} \rightarrow \infty$ for $t^{\prime}>0$

In the analysis the fluid considered to be optically thick, in addition to emission there is also selfabsorption and usually the absorption coefficient is wavelength dependent and larger. According to the Rosseland diffusion approximation for an optically thick fluid, the radiation heat flux is given by;

$q_{r}^{\prime}=-\frac{4 \sigma_{1}}{3 k_{1}} \frac{\partial T^{\prime 4}}{\partial y^{\prime}}$

where $\sigma_{1}\left(=5.67 \times 10^{-8}\right) \mathrm{W} / \mathrm{m}^{2} \mathrm{~K}^{4}$ is the Stefan-Boltzmann constant and $k_{1}\left(\mathrm{~m}^{-1}\right)$ is the Rosseland mean absorption coefficient. Assuming a small temperature difference between the fluid temperature $T^{\prime}$ and free stream temperature $T_{\infty}^{\prime}, T^{\prime}$ is expanded as a linear function about a free stream temperature $T_{\infty}^{\prime}$, after neglecting second and higher order terms in $\left(T^{\prime}-T_{\infty}^{\prime}\right)$, generates as a result of the form;

$T^{\prime 4} \cong 4 T_{\infty}^{\prime 3} T^{\prime}-3 T_{\infty}^{\prime 4}$

Using Eqs. (6) and (7), Eq. (3) reduces to;

$\rho C_{p} \frac{\partial T^{\prime}}{\partial t^{\prime}}=k \frac{\partial^{2} T^{\prime}}{\partial y^{\prime 2}}+\frac{16 \sigma_{1} T_{\infty}^{\prime 3}}{3 k_{1}} \frac{\partial^{2} T^{\prime}}{\partial y^{\prime 2}}+\frac{\rho D_{M} K_{T}}{C_{s}} \frac{\partial^{2} C^{\prime}}{\partial y^{\prime 2}}$ 
We introduce the following non-dimensional quantities;

$$
\begin{aligned}
& u=\frac{u^{\prime}}{U_{0}}, w=\frac{w^{\prime}}{U_{0}}, \eta=\frac{y^{\prime} U_{0}}{v}, \tau=\frac{t^{\prime} U_{0}^{2}}{v}, U=\frac{U_{0}^{3}}{v}, S_{c}=\frac{v}{D_{M}}, P_{r}=\frac{\mu C_{p}}{k}, K^{2}=\frac{v \Omega}{U_{0}^{2}}, \\
& \theta=\frac{T^{\prime}-T_{\infty}^{\prime}}{T_{w}^{\prime}-T_{\infty}^{\prime}}, \phi=\frac{C^{\prime}-C_{\infty}^{\prime}}{C_{w}^{\prime}-C_{\infty}^{\prime}}, \gamma=\frac{v K_{r}^{\prime}}{U_{0}^{2}}, N=\frac{k k_{1}}{4 \sigma_{1} T_{\infty}^{\prime 3}}, M^{2}=\frac{\sigma B_{0}{ }^{2} v}{\rho U_{0}^{2}}, K=\frac{K^{\prime} U_{0}^{2}}{v^{2}} \\
& D_{u}=\frac{D_{M} K_{T}\left(C_{w}^{\prime}-C_{\infty}^{\prime}\right)}{v T_{m}\left(T_{w}^{\prime}-T_{\infty}^{\prime}\right)}, G_{r}=\frac{g \beta_{\theta} v\left(T_{w}^{\prime}-T_{\infty}^{\prime}\right)}{U_{0}^{3}}, G_{m}=\frac{g \beta_{c} v\left(C_{w}^{\prime}-C_{\infty}^{\prime}\right)}{U_{0}^{3}}, S_{r}=\frac{D_{M} K_{T}\left(T_{w}^{\prime}-T_{\infty}^{\prime}\right)}{v T_{m}\left(C_{w}^{\prime}-C_{\infty}^{\prime}\right)} .
\end{aligned}
$$

where $G_{r}, G_{m}, M, m, K, K^{2}, P_{r}, N, D_{u}, S_{c}, \gamma$ and $S_{r}$ are respectively, the Thermal Grashof number, the mass Grashof number, the magnetic parameter, the Hall parameter, the permeability parameter, the rotation parameter, the Prandtl number, the radiation parameter, the Dufour number, the Schmidt number, the chemical reaction parameter and the Soret number.

We use Eq. (9) into Eqs. (1), (2), (4) and (8) the transformed governing coupled non-dimensional boundary layer equations of the flow become;

$$
\begin{aligned}
& \frac{\partial u}{\partial \tau}+2 K^{2} w=\frac{\partial^{2} u}{\partial \eta^{2}}-\frac{M^{2}}{\left(1+m^{2}\right)}(u+m w)+G_{r} \theta+G_{m} \phi-\frac{u}{K} \\
& \frac{\partial w}{\partial \tau}-2 K^{2} u=\frac{\partial^{2} w}{\partial \eta^{2}}+\frac{M^{2}}{\left(1+m^{2}\right)}(m u-w)-\frac{w}{K} \\
& \frac{\partial \theta}{\partial \tau}=\frac{1}{P_{r}}\left(1+\frac{4}{3 N}\right) \frac{\partial^{2} \theta}{\partial \eta^{2}}+D_{u} \frac{\partial^{2} \phi}{\partial \eta^{2}} \\
& \frac{\partial \phi}{\partial \tau}=\frac{1}{S_{c}} \frac{\partial^{2} \phi}{\partial \eta^{2}}+S_{r} \frac{\partial^{2} \theta}{\partial \eta^{2}}-\gamma \phi
\end{aligned}
$$

The initial and boundary conditions Eq. (5), using Eq. (9) is transformed to;

$$
\begin{array}{ll}
\tau \leq 0 ; u=0, w=0, \theta=0, \phi=0 & \forall \eta \\
\tau>0 ; u=\tau, w=0, \theta=1, \phi=1 & \text { at } \eta=0 \\
u \rightarrow 0, w \rightarrow 0, \theta \rightarrow 0, \phi \rightarrow 0 & \text { as } \eta \rightarrow \infty
\end{array}
$$

Skin-friction at the plate $(\eta=0)$ in $x$ and $z$-directions are given by;

$$
\tau_{x}=\left.\frac{\partial u}{\partial \eta}\right|_{\eta=0} \text { and } \tau_{z}=\left.\frac{\partial w}{\partial \eta}\right|_{\eta=0}
$$

The rate of heat and mass transfer in terms of Nusselt and Sherwood numbers, respectively at the plate $(\eta=0)$ are given by;

$$
N_{u}=-\left.\frac{\partial \theta}{\partial \eta}\right|_{\eta=0} \text { and } S_{h}=-\left.\frac{\partial \phi}{\partial \eta}\right|_{\eta=0}
$$


The transformed coupled partial differential equations describing the flow Eqs. (10) - (13) are highly non-linear and therefore, analytical solutions are not feasible. Hence, these equations together, with initial and boundary conditions Eq. (14) have been solved by employing a robust finite element method. The finite element method is a powerful computational technique that can be applied to solve ordinary and partial differential equations in a wide range of science and engineering problems. A comprehensive review of the finite element method is given in Reddy [33], Bathe [34] and Singiresu [35]. The variational form associated with Eqs. (10) - (13) over a typical 2 nodded linear element $\left(\eta_{e}, \eta_{e+1}\right)$ is given by;

$$
\begin{aligned}
& \int_{\eta_{e}}^{\eta_{e+1}} \psi_{1}\left[\frac{\partial u}{\partial \tau}-\frac{\partial^{2} u}{\partial \eta^{2}}+2 K^{2} w+\frac{M^{2}}{\left(1+m^{2}\right)}(u+m w)-\left(G_{r} \theta+G_{m} \phi\right)+\frac{u}{K}\right] d \eta=0 \\
& \int_{\eta_{e}}^{\eta_{e+1}} \psi_{2}\left[\frac{\partial w}{\partial \tau}-\frac{\partial^{2} w}{\partial \eta^{2}}-2 K^{2} u-\frac{M^{2}}{\left(1+m^{2}\right)}(m u-w)+\frac{w}{K}\right] d \eta=0 \\
& \int_{\eta_{e}}^{\eta_{e+1}} \psi_{3}\left[P_{r} \frac{\partial \theta}{\partial \tau}-\left(1+\frac{4}{3 N}\right) \frac{\partial^{2} \theta}{\partial \eta^{2}}-P_{r} D_{u} \frac{\partial^{2} \phi}{\partial \eta^{2}}\right] d \eta=0 \\
& \int_{\eta_{e+1}}^{\eta_{e}} \psi_{4}\left[S_{c} \frac{\partial \phi}{\partial \tau}-\frac{\partial^{2} \phi}{\partial \eta^{2}}-S_{c} S_{r} \frac{\partial^{2} \theta}{\partial \eta^{2}}-\gamma S_{c} \phi\right] d \eta=0
\end{aligned}
$$

where $\psi_{1}, \psi_{2}, \psi_{3}$ and $\psi_{4}$ are arbitrary test functions and may be viewed as the variations in $u, w, \theta$ and $\phi$, respectively. Integrating the second term in Eqs. (15) - (18) to reduce the order of integration, we obtain;

$$
\begin{aligned}
& \int_{\eta_{e}}^{\eta_{e+1}}\left[\begin{array}{l}
\left.\psi_{1}\left(\frac{\partial u}{\partial \tau}\right)-\left(\frac{\partial \psi_{1}}{\partial \eta}\right)\left(\frac{\partial u}{\partial \eta}\right)+\left(2 K^{2} w\right) \psi_{1}+\frac{M}{\left(1+m^{2}\right)}(u+m w) \psi_{1}\right] d \eta-\left[\psi_{1}\left(\frac{\partial u}{\partial \eta}\right)\right]_{\eta_{e}}^{\eta_{e+1}}=0 \\
-\left(G_{r} \theta+G_{m} \phi\right) \psi_{1}
\end{array}\right. \\
& \int_{\eta_{e}}^{\eta_{e+1}}\left[\psi_{2}\left(\frac{\partial w}{\partial \tau}\right)-\left(\frac{\partial \psi_{2}}{\partial \eta}\right)\left(\frac{\partial w}{\partial \eta}\right)-\left(2 K^{2} u\right) \psi_{2}+\frac{M}{\left(1+m^{2}\right)}(m u-w) \psi_{2}\right] d \eta-\left[\psi_{2}\left(\frac{\partial w}{\partial \eta}\right)\right]_{\eta_{e}}^{\eta_{e+1}}=0 \\
& \int_{\eta_{e}}^{\eta_{e+1}}\left[\psi_{3}\left(P_{r} \frac{\partial \theta}{\partial \tau}\right)-\left(1+\frac{3}{4 N}\right)\left(\frac{\partial \psi_{3}}{\partial \eta}\right)\left(\frac{\partial \theta}{\partial \eta}\right)-\left(P_{r} D_{u} \frac{\partial^{2} \phi}{\partial \eta^{2}}\right) \psi_{3}\right] d \eta-\left[\psi_{3}\left(\frac{\partial \theta}{\partial \eta}\right)\right]_{\eta_{e}}^{\eta_{e+1}}=0 \\
& \int_{\eta_{e}}^{\eta_{e+1}}\left[\left(S_{c} \frac{\partial \phi}{\partial \tau}\right) \psi_{4}-\left(\frac{\partial \psi_{4}}{\partial \eta}\right)\left(\frac{\partial \phi}{\partial \eta}\right)-\left(S_{c} S_{r} \frac{\partial^{2} \theta}{\partial \eta^{2}}\right) \psi_{4}-\left(\gamma S_{c} \phi\right) \psi_{4}\right] d \eta-\left[\psi_{4}\left(\frac{\partial \phi}{\partial \eta}\right)\right]_{\eta_{e}}^{\eta_{e+1}}=0
\end{aligned}
$$


To approximate the unknown fields $u(\eta, \tau), w(\eta, \tau), \theta(\eta, \tau)$ and $\phi(\eta, \tau)$, we substitute the finite element approximations of the form;

$u=\sum_{j=1}^{2} u_{j}^{e} \zeta_{j}^{e} ; w=\sum_{j=1}^{2} w_{j}^{e} \zeta_{j}^{e} ; \theta=\sum_{j=1}^{2} \theta_{j}^{e} \zeta_{j}^{e} ; \phi=\sum_{j=1}^{2} \phi_{j}^{e} \zeta_{j}^{e}$

with $\psi_{1}=\psi_{2}=\psi_{3}=\psi_{4}=\zeta_{j}^{e}(j=1,2)$ where $u_{j}^{e}, w_{j}^{e}, \theta_{j}^{e}$ and $\phi_{j}^{e}$ are respectively primary velocity, secondary velocity, temperature and concentration at $j^{\text {th }}$ node of the $e^{\text {th }}$ element $\left(\eta_{e}, \eta_{e+1}\right)$ and $\zeta_{j}$ 's are the shape functions, which are defined over the element $\left(\eta_{e}, \eta_{e+1}\right)$ as;

$\zeta_{1}^{e}=\frac{\eta_{e+1}-\eta}{\eta_{e+1}-\eta_{e}}$ and $\zeta_{2}^{e}=\frac{\eta-\eta_{e}}{\eta_{e+1}-\eta_{e}}$

For the purpose of numerical computations, the whole domain is divided into a set of 80 line elements of equal width 0.05 , each element being two nodded. After some numerical experimentation, the boundary condition $\eta \rightarrow \infty$ has been fixed at $\eta_{\max }=4$, where $\eta_{\max }$ represents infinity $(\eta=\infty)$. The value greater than 4 does not result in any significant change in the numerical results. On using Eqs. (23) - (24) into Eqs. (19) - (22) after assembly of all the element equations by inter-element connectivity conditions, we obtain a matrix of order $324 \times 324$. This system of equations are extremely non-linear, so that an iterative scheme has been used to solve it. After using the given boundary conditions, a system of 316 equations remain for numerical solution and these equations are solved using Gauss elimination method by maintaining the desired accuracy 0.0005 .

Tables 1 and 2 presents the numerical values of primary velocity $u$, secondary velocity $w$, temperature $\theta$ and concentration $\phi$ for a variety of mesh sizes $0.025,0.05$ and 0.075 at $\tau=1.0$. It has been noticed that there is no change in the numerical values of $u, w, \theta$ and $\phi$ for different mesh sizes at $\tau=1.0$, which designates the results are mesh independent. Finally, in all the numerical computations the mesh size 0.05 was selected.

Table 1 Numerical values of $\mathrm{u}$ and $\mathrm{w}$ for various mesh sizes at $\tau=1.0$.

\begin{tabular}{cccccc}
\hline \multicolumn{2}{c}{ Mesh size $=\mathbf{0 . 0 2 5}$} & \multicolumn{2}{c}{ Mesh size $=\mathbf{0 . 0 5 0}$} & \multicolumn{2}{c}{ Mesh size $=\mathbf{0 . 0 7 5}$} \\
\hline$u$ & $w$ & $u$ & $w$ & $u$ & $w$ \\
\hline 1.000000 & 0.000000 & 1.000000 & 0.000000 & 1.000000 & 0.000000 \\
0.571295 & 0.656525 & 0.571295 & 0.656525 & 0.571295 & 0.656525 \\
0.352849 & 0.482914 & 0.352849 & 0.482914 & 0.352849 & 0.482914 \\
0.222090 & 0.291166 & 0.222090 & 0.291166 & 0.222090 & 0.291166 \\
0.134710 & 0.162978 & 0.134710 & 0.162978 & 0.134710 & 0.162978 \\
0.077649 & 0.086520 & 0.077649 & 0.086520 & 0.077649 & 0.086520 \\
0.042540 & 0.043815 & 0.042540 & 0.043815 & 0.042540 & 0.043815 \\
0.022136 & 0.021180 & 0.022136 & 0.021180 & 0.022136 & 0.021180 \\
0.010745 & 0.009631 & 0.010745 & 0.009631 & 0.010745 & 0.009631 \\
0.004314 & 0.003686 & 0.004314 & 0.003686 & 0.004314 & 0.003686 \\
0.000000 & 0.000000 & 0.000000 & 0.000000 & 0.000000 & 0.000000 \\
\hline
\end{tabular}


Table 2 Numerical values of $\theta$ and $\phi$ for various mesh sizes at $\tau=1.0$.

\begin{tabular}{cccccc}
\hline \multicolumn{2}{c}{ Mesh size $=\mathbf{0 . 0 2 5}$} & \multicolumn{2}{c}{ Mesh size $=\mathbf{0 . 0 5 0}$} & \multicolumn{2}{c}{ Mesh size $=\mathbf{0 . 0 7 5}$} \\
\hline$\theta$ & $\phi$ & $\theta$ & $\phi$ & $\theta$ & $\phi$ \\
\hline 1.000000 & 1.000000 & 1.000000 & 1.000000 & 1.000000 & 1.000000 \\
0.707433 & 0.765487 & 0.707433 & 0.765487 & 0.707433 & 0.765487 \\
0.455141 & 0.572883 & 0.455141 & 0.572883 & 0.455141 & 0.572883 \\
0.265877 & 0.414002 & 0.265877 & 0.414002 & 0.265877 & 0.414002 \\
0.141287 & 0.285768 & 0.141287 & 0.285768 & 0.141287 & 0.285768 \\
0.068599 & 0.187007 & 0.068599 & 0.187007 & 0.068599 & 0.187007 \\
0.030619 & 0.115488 & 0.030619 & 0.115488 & 0.030619 & 0.115488 \\
0.012648 & 0.066906 & 0.012648 & 0.066906 & 0.012648 & 0.066906 \\
0.004836 & 0.035504 & 0.004836 & 0.035504 & 0.004836 & 0.035504 \\
0.001588 & 0.015151 & 0.001588 & 0.015151 & 0.001588 & 0.015151 \\
0.000000 & 0.000000 & 0.000000 & 0.000000 & 0.000000 & 0.000000 \\
\hline
\end{tabular}

\section{Results and discussion}

In order to determine the physical insight of the problem, the effects of various flow controlled parameters such as $G_{r}, G_{m}, K^{2}, M^{2}, m, K, P_{r}, N, D_{u}, S_{c}, S_{r}$ and $\gamma$ on the primary velocity $u$, secondary velocity $w$, fluid temperature $\theta$, fluid concentration $\phi$ are displayed graphically in Figures 2 - 19. Numerical values of primary and secondary skin-frictions $\tau_{x}$ and $\tau_{z}$, Nusselt number $N_{u}$ and Sherwood number $S_{h}$ at the plate are presented through the Tables 3 - 6. Prandtl number $P_{r}$ and Schmidt number $S_{c}$ are chosen as $0.3 \leq P_{r} \leq 1.0$ and $0.22 \leq S_{c} \leq 1.0$. These are the most encountered fluids used in plasma physics, engineering and industries. In the entire computation procedure, the thermo-physical parameters specified as $\quad G_{r}=5, G_{m}=5, P_{r}=0.71, S_{c}=0.22, N=2, m=0.5, M=12, D_{u}=0.03, S_{r}=1$, $K=0.5, \gamma=1, K^{2}=2$ and $\tau=1$.

Figures 2 and 3 illustrate the effects of thermal and mass Grashof numbers on the primary and secondary velocity components $u$ and $w$ respectively. It is observed that both primary and secondary velocity components enhanced with an increase in thermal Grashof number $G_{r}$ and mass Grashof number $G_{m}$. An increase in $G_{r}$ leads to decrease of drag force and hence increase in the velocity profiles in the boundary layer. As mass Grashof number $G_{m}$ increases, decrease viscous hydrodynamic force as a result momentum of the fluid is higher. Also it is noticed that velocity components rise suddenly near the plate and after this fluid velocity components asymptotically decreases to zero as $\eta \rightarrow \infty$. This trend is clearly supported by the physical reality, since the buoyancy effects are significant near the plate, which results a suddenly lift up of the velocity components near to the plate. The primary and secondary velocity components $u$ and $w$ decreases within the boundary layer with an increase in the magnetic parameter $M$ as shown in Figure 4. As the strength of the magnetic field increase, the Lorentz force associated with the magnetic field makes the boundary layer thicker as a result the momentum of the fluid velocity components decreases. Figure 5 elucidates the influence of Hall parameter $m$ on the primary and secondary velocities $u$ and $w$. It is seen that an increase in the Hall parameter tends to accelerate both primary and secondary velocity components throughout the boundary layer region since Hall current induces a secondary flow in the flow domain that plays a vital role in describing the flow field characteristics. The effects of rotation parameter $K^{2}$ on the primary velocity $u$ and secondary velocity $w$ are depicted in Figure 6. It is perceived that an increase in the rotation parameter tends to decline the primary velocity $u$ whereas the reverse effect is noticed on the secondary velocity $w$. This implies that rotation has a tendency that retards the fluid flow in the primary flow direction and accelerates the fluid flow in the secondary flow direction which is endorsed to the fact that when the frictional layer at the moving plate is suddenly set into the motion then the Coriolis force acts as a constraint in the main flow direction to generate the secondary flow. Figure 7 illustrates the influence of the permeability parameter $K$ on primary and secondary velocity components $u$ and $w$. It is clear that both $u$ and $w$ increases 
throughout the boundary layer region with increment in the permeability parameter due to decrease of drag force on the boundary layer. The effect of Prandtl number $P_{r}$ on the primary velocity $u$, secondary velocity $w$ and temperature $\theta$ are plotted in Figures $\mathbf{8}$ and $\mathbf{9}$, respectively. It is observed that both primary and secondary velocity components and temperature decreases throughout the boundary layer as the Prandtl number increases. The Prandtl number $P_{r}$ is the ratio of momentum to thermal diffusivity. An increase in the Prandtl number causes to significant reduction in the momentum and thermal boundary layer thickness, as a result fluid velocity components and temperature decreases throughout the boundary layer. Figures 10 and 11 plotted to show the impact of radiation parameter $N$ on the primary velocity $u$, secondary velocity $w$ and temperature $\theta$, respectively. It is clear that an increase in the thermal radiation tends to decrease in each of the fluid velocity components and temperature. Physically, thermal radiation causes to fall in the fluid temperature and thereby causes to fall in the kinetic energy of the fluid particles. This result is an excellent agreement with the laws of physics.

The effects of the Dufour number $D_{u}$ on the primary velocity $u$, secondary velocity $w$ and temperature $\theta$ are depicted in Figures $\mathbf{1 2}$ and 13, respectively. It is noticed that increasing values of $D_{u}$ tends to enhance each of the fluid velocity components and fluid temperature in the boundary layer. Physically, Dufour number produces heat in the fluid flow that measures the concentration gradient to thermal energy flux in the flow field. Figures 14 and 15 show the effects of Schmidt number $S_{c}$ on the primary velocity $u$, secondary velocity $w$ and concentration $\phi$, respectively. It is observed that $u, w$ and $\phi$ decrease throughout the boundary layer with increasing of $S_{c}$. As the Schmidt number increase the species concentration decrease causes the concentration buoyancy effects to decrease yielding a reduction in the fluid velocity components. Figures $\mathbf{1 6}$ and $\mathbf{1 7}$ demonstrate the impact of Soret number $S_{r}$ on the primary velocity $u$, secondary velocity $w$ and concentration $\phi$, respectively. It is observed that $u, w$ and $\phi$ increased in the boundary layer as the Soret number $S_{r}$ increased due to mass flux created by temperature gradients. Figures 18 and 19 represent the effects of chemical reaction parameter $\gamma$ on $u, w$ and $\phi$, respectively. It can be seen that $u, w$ and $\phi$ are diminished in the boundary layer as the chemical reaction parameter increased due to decrease of momentum and concentration boundary layer thickness.

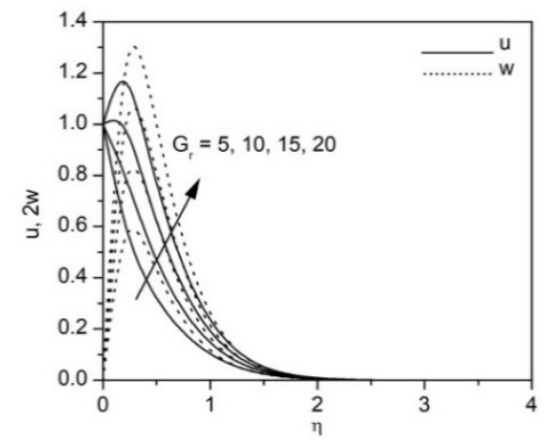

Figure 2 Variation of the primary and secondary velocity profiles with different $G_{r}$.

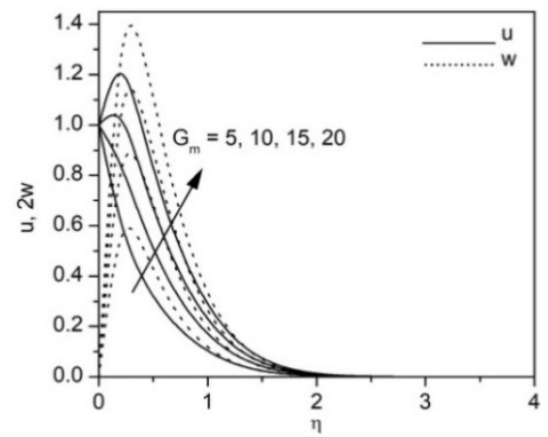

Figure 3 Variation of the primary and secondary velocity profiles with different $G_{m}$. 


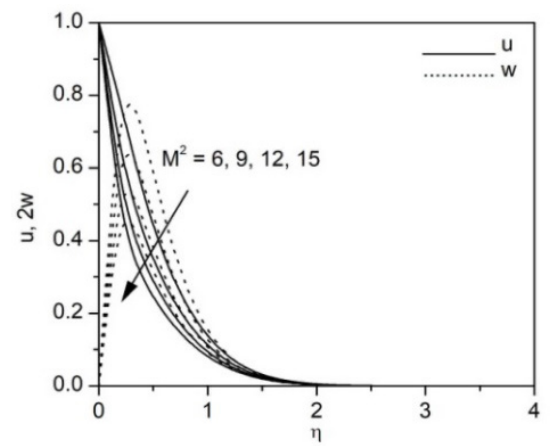

Figure 4 Variation of the primary and secondary velocity profiles with different $M^{2}$.

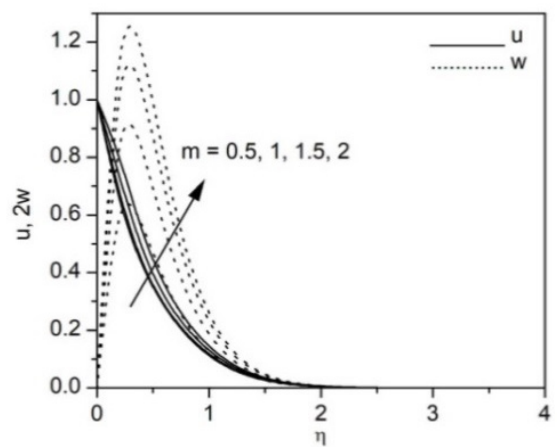

Figure 5 Variation of the primary and secondary velocity profiles with different $\mathrm{m}$.

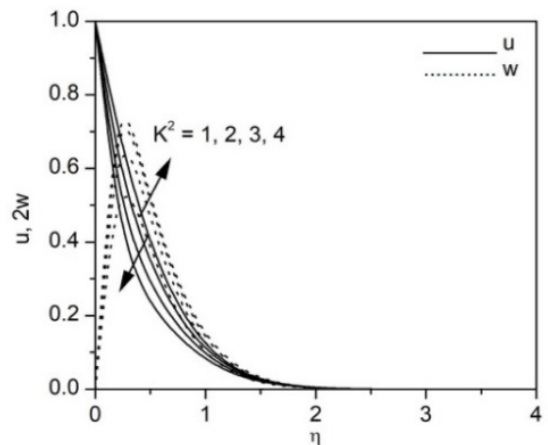

Figure 6 Variation of the primary and secondary velocity profiles with different $K^{2}$.

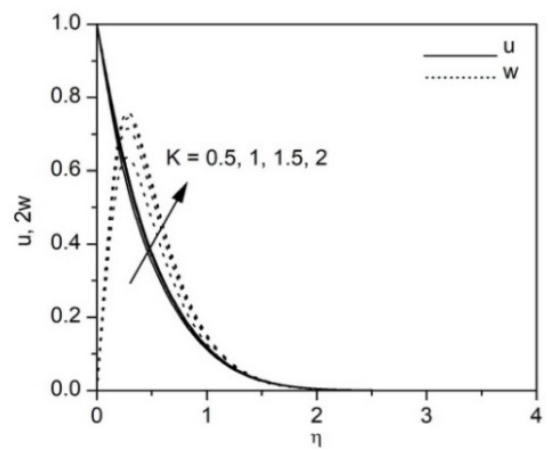

Figure 7 Variation of the primary and secondary velocity profiles with different $\mathrm{K}$. 


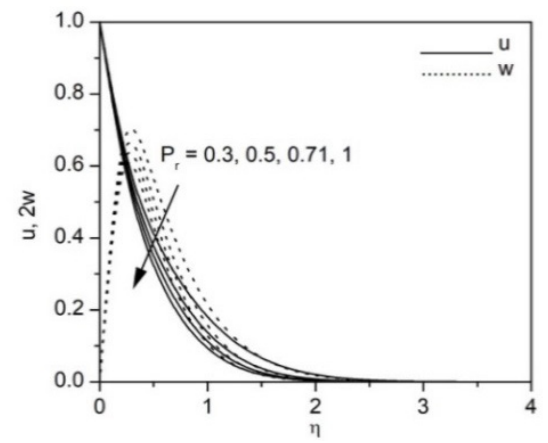

Figure 8 Variation of the primary and secondary velocity profiles with different $P_{r}$.

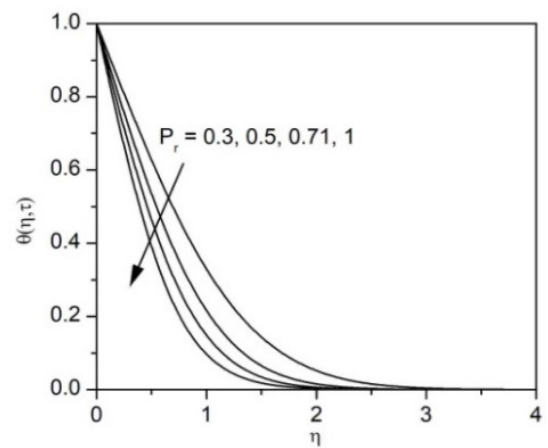

Figure 9 Variation of the temperature profiles with different $P_{r}$.

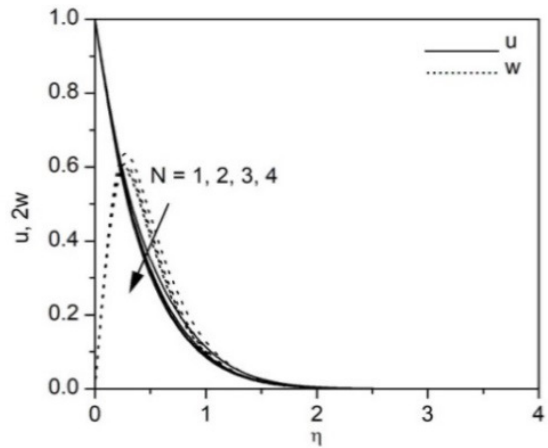

Figure 10 Variation of the primary and secondary velocity profiles with different $\mathrm{N}$.

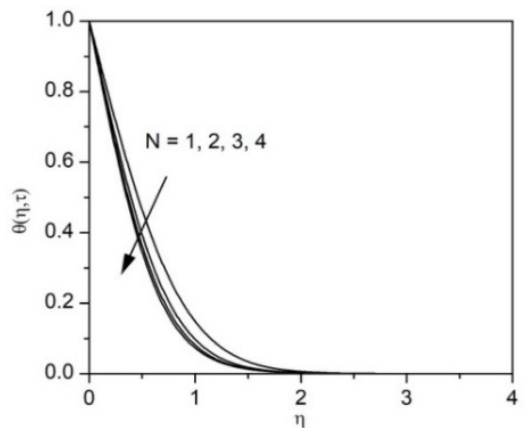

Figure 11 Variation of the temperature profiles with different N. 


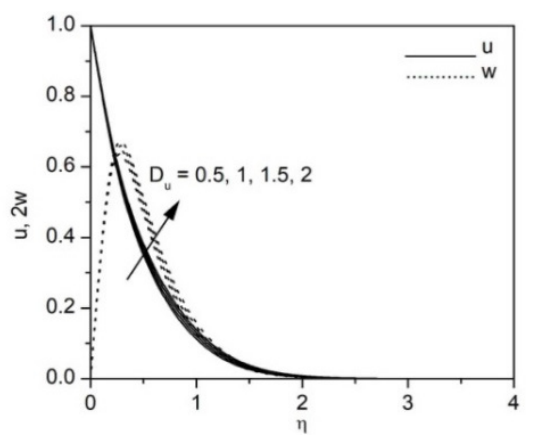

Figure 12 Variation of the primary and secondary velocity profiles with different $D_{u}$.

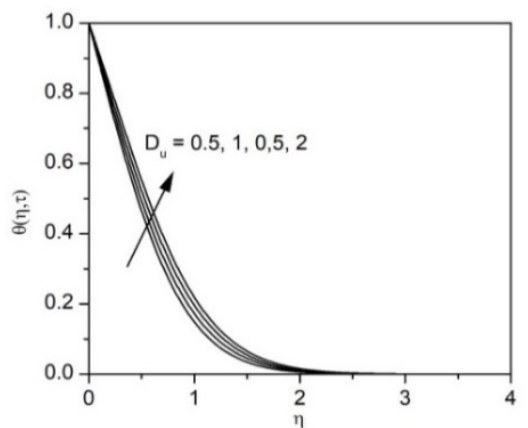

Figure 13 Variation of the temperature profiles with different $D_{u}$.

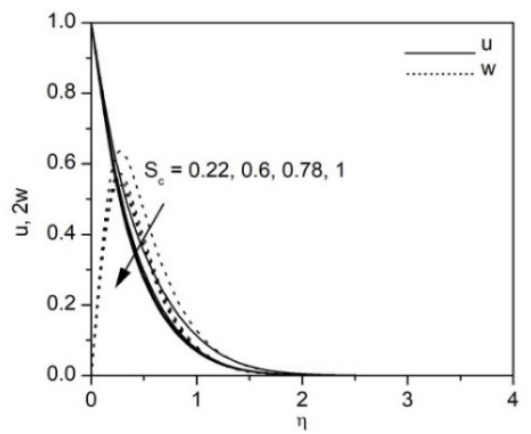

Figure 14 Variation of the primary and secondary velocity profiles with different $S_{c}$.

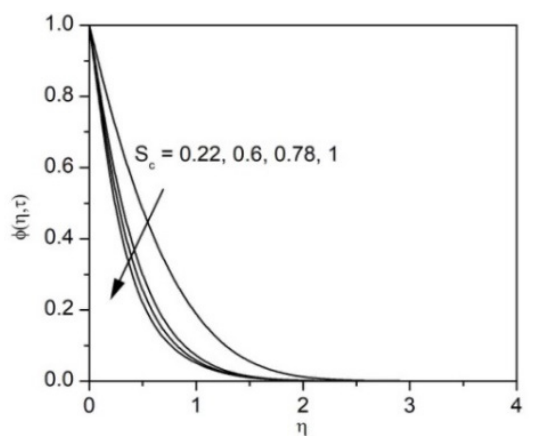

Figure 15 Variation of the concentration profiles with different $S_{c}$. 


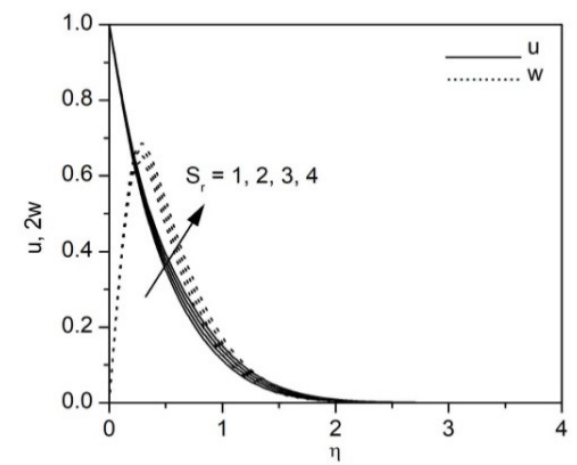

Figure 16 Variation of the primary and secondary velocity profiles with different $S_{r}$.

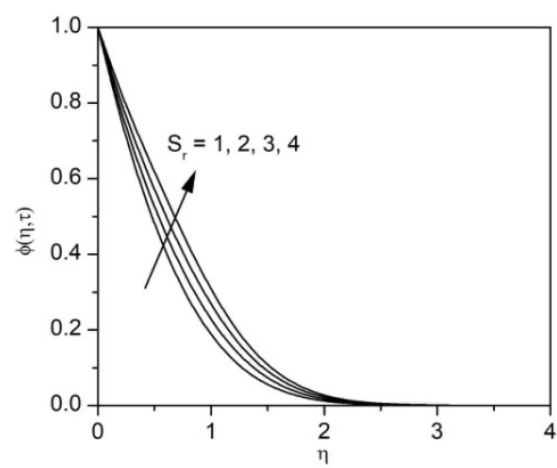

Figure 17 Variation of the concentration profiles with different $S_{r}$.

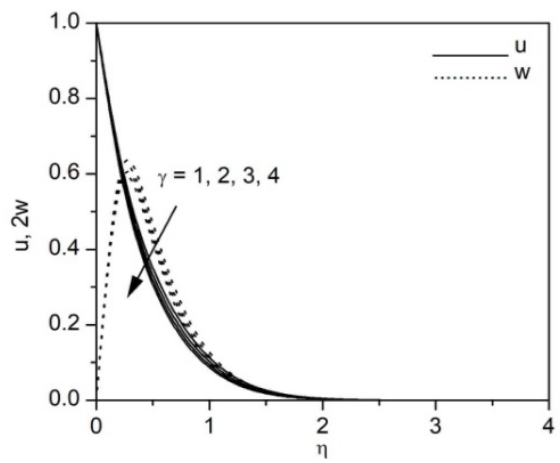

Figure 18 Variation of the primary and secondary velocity profiles with different $\gamma$.

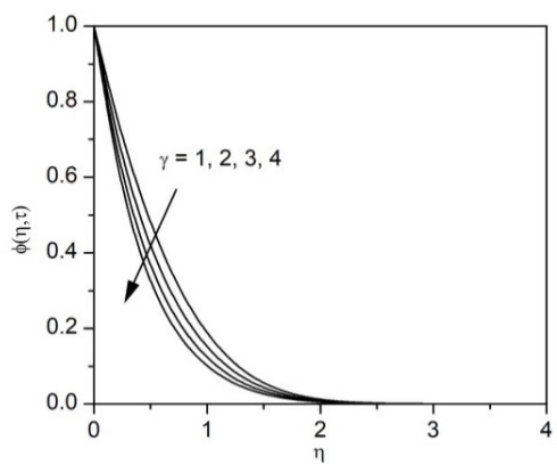

Figure 19 Variation of the concentration profiles with different $\gamma$. 
Tables 3 and 4 displays the variation of the primary skin friction $\left(\tau_{x}\right)$ and secondary skin friction $\left(\tau_{z}\right)$ under the influence of $G_{r}, G_{m}, K, M^{2}, m, K^{2}, P_{r}, N, D_{u}, S_{c}, S_{r}$ and $\gamma$. Tables 3 and 4 reveals that both primary and secondary skin frictions tends to increase with increasing values of $G_{r}, G_{m}, m, K, D_{u}$ and $S_{r}$ while both tends to decrease with increasing values of $M^{2}, P_{r}, N, S_{c}$ and $\gamma$. As rotational parameter $K^{2}$ increase primary skin friction tends to increase and reverse trend is noticed on the secondary skin friction. Table 5 shows the variation of Nusselt number $N_{u}$ under the influence of $P_{r}, N$ and $D_{u}$. From Table 5, it is observed that an increase in $P_{r}$ and $N$ leads to enhance Nusselt number while it decreases when $D_{u}$ increases. Table 6 indicates the variation of Sherwood number $S_{h}$ under the influence of $S_{c}, S_{r}$ and $\gamma$. It is noticed from Table 6 that Sherwood number increases with increasing $S_{c}$ and $\gamma$ while it decreases with increasing $S_{r}$.

Table 3 Variation of skin friction $\tau_{\mathrm{x}}$ and $\tau_{\mathrm{z}}$ at the plate $(\eta=0)$.

\begin{tabular}{|c|c|c|c|c|c|c|c|}
\hline$P_{r}$ & $G_{r}$ & $G_{m}$ & $M^{2}$ & $m$ & $K^{2}$ & $\tau_{x}$ & $\tau_{z}$ \\
\hline 0.3 & & & & & & 2.654032 & 1.574260 \\
\hline 0.5 & 5 & 5 & 9 & 0.5 & 2 & 2.589600 & 1.516520 \\
\hline 0.71 & & & & & & 2.535856 & 1.471394 \\
\hline \multirow{3}{*}{0.71} & 5 & \multirow{3}{*}{5} & \multirow{3}{*}{9} & \multirow{3}{*}{0.5} & \multirow{3}{*}{2} & 2.535856 & 1.471394 \\
\hline & 10 & & & & & 3.259632 & 1.887720 \\
\hline & 15 & & & & & 4.164352 & 2.408126 \\
\hline \multirow{3}{*}{0.71} & \multirow{3}{*}{5} & 5 & \multirow{3}{*}{9} & \multirow{3}{*}{0.5} & \multirow{3}{*}{2} & 2.535856 & 1.471394 \\
\hline & & 10 & & & & 3.439072 & 2.007054 \\
\hline & & 15 & & & & 4.342284 & 2.549912 \\
\hline \multirow{3}{*}{0.71} & \multirow{3}{*}{5} & \multirow{3}{*}{5} & 6 & \multirow{3}{*}{0.5} & \multirow{3}{*}{2} & 3.125656 & 1.748458 \\
\hline & & & 9 & & & 2.535856 & 1.471394 \\
\hline & & & 12 & & & 2.109336 & 1.249556 \\
\hline \multirow{3}{*}{0.71} & \multirow{3}{*}{5} & \multirow{3}{*}{5} & & 0.5 & \multirow{3}{*}{2} & 2.535856 & 1.471394 \\
\hline & & & 9 & 1.0 & & 2.655728 & 2.079840 \\
\hline & & & & 1.5 & & 2.935172 & 2.506322 \\
\hline \multirow{3}{*}{0.71} & \multirow{3}{*}{5} & \multirow{3}{*}{5} & & \multirow{3}{*}{0.5} & 1 & 2.857576 & 1.201624 \\
\hline & & & 9 & & 2 & 2.535856 & 1.471394 \\
\hline & & & & & 3 & 2.193728 & 1.636104 \\
\hline
\end{tabular}

Table 4 Variation of skin friction $\tau_{x}$ and $\tau_{z}$ at the plate $(\eta=0)$.

\begin{tabular}{|c|c|c|c|c|c|c|c|}
\hline$K$ & $S_{c}$ & $N$ & $D_{u}$ & $S_{r}$ & $\gamma$ & $\tau_{x}$ & $\tau_{z}$ \\
\hline 0.5 & & & & & & 2.535856 & 1.471394 \\
\hline 1.0 & 0.22 & 1 & 0.5 & 1 & 1 & 2.660200 & 1.640246 \\
\hline 1.5 & & & & & & 2.702716 & 1.702008 \\
\hline \multirow{3}{*}{0.5} & 0.22 & \multirow{3}{*}{1} & \multirow{3}{*}{0.5} & \multirow{3}{*}{1} & \multirow{3}{*}{1} & 2.535856 & 1.471394 \\
\hline & 0.60 & & & & & 2.384656 & 1.358896 \\
\hline & 0.78 & & & & & 2.336124 & 1.326646 \\
\hline \multirow{3}{*}{0.5} & \multirow{3}{*}{0.22} & 1 & \multirow{3}{*}{0.5} & \multirow{3}{*}{1} & \multirow{3}{*}{1} & 2.535856 & 1.471394 \\
\hline & & 2 & & & & 2.475924 & 1.424078 \\
\hline & & 3 & & & & 2.447764 & 1.402866 \\
\hline \multirow{3}{*}{0.5} & \multirow{3}{*}{0.22} & \multirow{3}{*}{1} & 0.5 & \multirow{3}{*}{1} & \multirow{3}{*}{1} & 2.535856 & 1.471394 \\
\hline & & & 1.0 & & & 2.560296 & 1.489862 \\
\hline & & & 1.5 & & & 2.585636 & 1.508890 \\
\hline \multirow{3}{*}{0.5} & \multirow{3}{*}{0.22} & \multirow{3}{*}{1} & \multirow{3}{*}{0.5} & 1 & \multirow{3}{*}{1} & 2.535856 & 1.471394 \\
\hline & & & & 2 & & 2.564484 & 1.496418 \\
\hline & & & & 3 & & 2.594284 & 1.522244 \\
\hline \multirow{3}{*}{0.5} & \multirow{3}{*}{0.22} & \multirow{3}{*}{1} & \multirow{3}{*}{0.5} & \multirow{3}{*}{1} & 1 & 2.535856 & 1.471394 \\
\hline & & & & & 2 & 2.482876 & 1.441598 \\
\hline & & & & & 3 & 2.436768 & 1.415156 \\
\hline
\end{tabular}


Table 5 Variation of Nusselt number $\mathrm{N}_{\mathrm{u}}$ at the plate $(\eta=0)$.

\begin{tabular}{cccc}
\hline$P_{r}$ & $N$ & $D_{u}$ & $N_{u}$ \\
\hline $\mathbf{0 . 3}$ & 1 & 0.5 & 0.922968 \\
$\mathbf{0 . 5}$ & & & 1.179420 \\
$\mathbf{0 . 7 1}$ & 1 & 0.5 & 1.395738 \\
\hline & 2 & & 1.395738 \\
$\mathbf{0 . 7 1}$ & 3 & 0.5 & 1.640922 \\
& 1 & 1.0 & 1.757976 \\
\hline & & 1.5 & 1.295738 \\
$\mathbf{0 . 7 1}$ & & & 1.1800236 \\
\hline
\end{tabular}

Table 6 Variation of Sherwood number $S_{h}$ at the plate $(\eta=0)$.

\begin{tabular}{cccc}
\hline$S_{c}$ & $S_{r}$ & $\gamma$ & $S_{h}$ \\
\hline $\mathbf{0 . 2 2}$ & 1 & 1 & 1.462932 \\
$\mathbf{0 . 6 0}$ & & & 1.883015 \\
$\mathbf{0 . 7 8}$ & 1 & 1 & 2.105060 \\
\hline & 2 & & 1.462932 \\
$\mathbf{0 . 2 2}$ & 3 & 1 & 1.104195 \\
& 1 & 2 & 0.984300 \\
\hline & & 3 & 1.462932 \\
$\mathbf{0 . 2 2}$ & & & 1.466590 \\
& & & \\
\hline
\end{tabular}

\section{Conclusions}

In the present work, we have examined the governing equations for the effects of Hall current and rotation MHD chemically reacting and radiating flow past an accelerated vertical plate through the porous medium in the presence of Soret and Dufour effects numerically by finite element method. We conclude that thermal and mass buoyancy forces tend to improve both primary and secondary fluid velocities whereas magnetic parameter and radiation have reverse trend. The fluid rotation tends to retard the flow in the primary flow direction and it helps to accelerate the flow in the secondary flow direction. Thermal diffusion and diffusion thermo effects support to enhance both velocity components, whereas chemical reaction rate tends to depreciate both velocity components. Hall current helps to develop both velocity components. These parameters have similar influence on the primary and secondary skin frictions. Increase in the radiation parameter leads to reduce fluid temperature whereas diffusion thermo effect has opposite effect and these parameters have opposite effect on the Nusselt number. Thermal diffusion effects tend to increase the species concentration whereas reverse trend was observed with increase in chemical reaction rate and these parameters have opposite effect on Sherwood number. Adequate knowledge of this model are useful in geophysical energy systems, hydromagnetic flow of conducting transport in packed beds, oil extraction, rotating MHD generators, biological sciences and refrigeration coils.

\section{Acknowledgements}

The authors are thankful to the management of the University of Dodoma, Tanzania, for their encouragement. We would also extend thanks to the editor and the reviewers for their thoughtful comments toward improving our manuscript. 


\section{References}

[1] OD Makinde. MHD mixed convection interaction with Thermal radiation and nth order chemical reaction past a vertical porous. Chem. Eng. Commun. 2010; 198, 37- 41.

[2] MG Reddy. Thermal radiation and chemical reaction effects on MHD mixed convective boundary layer slip flow in a porous medium with heat source and Ohmic heating. Eur. Phys. J. Plus 2014; 129, 41.

[3] J Manjula, P Padma, MG Reddy and M Venakateswarlu. Influence of thermal radiation and chemical reaction on MHD flow, heat and mass transfer over a stretching surface. Proc. Eng. 2015; 127, 1315-22.

[4] M Venakateswarlu and P Padma. Unsteady MHD free convection heat and mass transfer in a boundary layer flow past a vertical permeable plate with thermal radiation and chemical reaction. Proc. Eng. 2015; 127, 791-9.

[5] CS Lakshmi and R Muthucumaraswamy. Thermal radiation and chemical reaction effects on exponentially accelerated vertical plate with variable temperature and uniform mass diffusion. Int. J. Eng. Sci. Res. Tech. 2016, 5, 321-32.

[6] R Muthucumaraswamy and P Sivakumar. MHD flow past a paraboic flow past an infinite isothermal vertical plate in the presence of thermal radiation and chemical reaction. Int. J. Appl. Mech. Eng. 2016; 21, 95-105.

[7] AS Eegunjobi, OD Makinde and S Jangili. Unsteady MHD chemically reacting and radiating mixed convection slip flow past a stretching surface in a Porous medium. Defect Diffusion Forum 2017; 377, 200-10.

[8] BP Reddy. Radiation effects on unsteady MHD flow over a vertical plate with ramped wall temperature in the presence of chemical reaction. Int. J. Math. Arch. 2017; 8, 63-70.

[9] RS Babu, BR Kumar and OD Makinde. Chemical reaction and thermal radiation effects on MHD mixed convection over a vertical plate with variable fluid properties. Defect Diffusion Forum 2018; 387, 332-42.

[10] BP Reddy. Effects of chemical reaction on transient MHD flow with mass transfer past an impulsively fixed infinite verticalplate in the presence of thermal radiation. Int. J. Appl. Mech. Eng. 2019; 24, 169-82.

[11] AS Idowu and U Sani. Thermal radiation and chemical reaction effects on unsteady MHD third grade fluid flow between stationary and oscillating plates. Int. J. Appl. Meh. Eng. 2019; 24, 269-93.

[12] S Siddiqa, MA Hossain and RSR Gorla. Hall current effects on magnetohydrodynamic natural convection flow with strong cross magnetic field. Int. J. Ther. Sci. 2013; 71, 196-204.

[13] NN Anika and N Islam. Hall current effects on magneto-hydrodynamics fluid over an infinite rotating vertical porous plate embedded in unsteady laminar flow. Ann. Pure. Appl. Math. 2013; 3, 189-200.

[14] AN Maguna and NM Mutua. Hall current effects on free convection flow and mass transfer past a semi-infinite vertical flat plate. Int. J. Math. Stat. Stud. 2013; 1, 1-22.

[15] GS Seth, S Sarkar and SM Hussain. Effects of Hall current, radiation and rotation on natural convection heat and mass transfer flow past a moving vertical plate. Ain Shams Eng. J. 2014; 5, 489-509.

[16] JK Sundarnath and R Muthucumarswamy. Hall effects on MHD flow past an accelerated plate with heat transfer. Int. J. Appl. Mech. Eng. 2015; 20, 171-81.

[17] P Sulochana. Hall current effects on unsteady MHD convective flow of heat generating/absorbing fluid through porous medium in a rotating parallel plate channel. Int. J. Adv. Res. Ideas Innovat. Tech. 2016; 2, 1-11.

[18] US Rajput and N Kanaujia. MHD flow past a vertical plate with variable temperature and mass diffusion in the presence of Hall current, Int. J. Appl. Sci. Eng. 2016; 14, 115-23.

[19] GS Seth, A Bhattacharyya and R Tripathi. Effect of Hall current on MHD natural convection heat and mass transfer flow of rotating fluid past a vertical plate with ramped wall temperature. Front. Heat Mass Tran. 2017; 9, 21.

[20] GS Seth, B Kumbhakar and S Sarkar. Unsteady MHD natural convection flow with exponentially accelerated free-stream past a vertical plate in the presence of hall current and rotation. Rend. Circ. Mat. Palermo Ser. II 2017; 66, 263-83.

[21] KVC Sekhar. MHD free convective heat and mass transfer flow past an accelerated vertical plate through a porous medium with Hall current, rotation and Soret effects. Int. J. Mech. Prod. Eng. Res. Dev. 2018; 8, 685-706. 
[22] BP Reddy. Hall effect on MHD transient flow past an impulsively started infinite horizontal porous plate in a rotating system. Int. J. Appl. Mech. Eng. 2018; 23, 471-83.

[23] MV Krishna, MG Reddy and AJ Chamkha. Heat and mass transfer on MHD rotating flow of second grade fluid past an infinite vertical plate embedded in uniform porous medium with Hall effects. In: BR Kumar, R Sivaraj, BSRV Prasad, M Nalliah, AS Reddy (Eds.). Birkhäuser, Cham, 2019, p. 417-27.

[24] MV Krishna. Hall and ion slip effects on MHD free convective rotating flow bounded by the semiinfinite vertical porous surface. Heat Tran. 2020; 49, 1920-38.

[25] ERG Eckert and RM Drake. Analysis of heat and mass transfer. Mc. Graw-Hill, New York, 1972.

[26] OD Makinde. On MHD mixed convection with Soret and Dufour effects past a vertical plate embedded in porous medium. Lat. Am. Appl. Res. 2011; 41, 63-8.

[27] D Srinivasacharya and C Ramreddy. Soret and Dufour effects on mixed convection from an exponentially stretching surface. Int. J. Nonlinear Sci. 2011; 12, 60-8.

[28] MJ Subhakar and K Gangadhar. Soret and Dufour effects on MHD convective flow of heat and mass transfer over a moving non-isothermal vertical plate with heat generation/absorption. $A d v$. Appl. Sci. Res. 2012; 3, 3165-84.

[29] CY Cheng. Soret and Dufour effects on free convection heat and mass transfer from an arbitrarily inclined plate in a porous medium with constant wall temperature and concentration. Int. Commun. Heat Mass Trans. 2012; 39, 72-7.

[30] D Srinivasacharya and S Reddy. Soret and Dufour effects on mixed convection from a vertical plate in power law fluid saturated porous medium. Theor. Appl. Mech. 2013; 40, 525-42.

[31] YI Seini and OD Makinde. Hydromagnetic flow with Dufour and Soret effects past a vertical plate embedded in porous media. Math. Theor. Model. 2013; 3, 47-65.

[32] A Hussanan, MZ Salleh, I Khan, RM Tahar and Z Ismail. Soret effects on unsteady magnetohydrodynamic mixed convection heat and mass transfer flow in a porous medium with Newtonian heating. Maejo Int. J. Sci. Tech. 2015; 9, 224-45.

[33] JN Reddy. An introduction to the finite element methods. $2^{\text {nd }}$ ed. Mc Graw-Hill, NewYork, 1993.

[34] KJ Bathe. Finite element procedures. Prentice-Hall, New Jersey, 1996.

[35] SR Singiresu. The finite element methods in engineering. $5^{\text {th }}$ ed. Elsevier, Burlington, 2011. 